\section{Nogo-A-specific antibody treatment enhances sprouting and functional recovery after cervical lesion in adult primates}

\author{
Patrick Freund ${ }^{1,5}$, Eric Schmidlin ${ }^{1,5}$, Thierry Wannier ${ }^{1,2,5}$, \\ Jocelyne Bloch ${ }^{3}$, Anis Mir ${ }^{4}$, Martin E Schwab ${ }^{2}$ \& \\ Eric M Rouiller ${ }^{1}$ \\ In rodents, after spinal lesion, neutralizing the neurite growth \\ inhibitor Nogo-A promotes axonal sprouting and functional \\ recovery. To evaluate this treatment in primates, 12 monkeys \\ were subjected to cervical lesion. Recovery of manual dexterity \\ and sprouting of corticospinal axons were enhanced in monkeys \\ treated with Nogo-A-specific antibody as compared to monkeys \\ treated with control antibody.
}

After injury, the adult mammalian central nervous system has only a limited capacity to repair itself. Transected nerve fibers do not spontaneously regrow, in part because of the effects of myelinassociated neurite growth inhibitors such as Nogo- $\mathrm{A}^{1,2}$. After section of the corticospinal tract in adult rats, neutralizing Nogo-A with monoclonal antibodies leads to enhancement of axonal regrowth and compensatory sprouting, accompanied by increased motor recovery ${ }^{2-4}$. The neutralization of Nogo-A represents a promising approach for therapy after lesion if its efficacy can be demonstrated in primates. First, the general organization of the human corticospinal system is similar to that of monkeys but not to that of rats ${ }^{5}$. Second, the corticospinal system is involved in dexterous hand movements typical of primates ${ }^{6-8}$. Behavioral assessment of manual dexterity is more pertinent in monkeys than rodents, the former being capable of dexterous movements of the fingers, as are humans. Third, neutralization of Nogo-A may not only favor sprouting of lesioned axons, but may also induce unspecific growth of axons, causing undesired pathologies. The monkey model offers more possibilities than rodents to detect such changes of behavior.

Neutralization of Nogo-A was found to promote sprouting of corticospinal axons in marmosets 9 . The aim of our study was to extend these anatomical data to macaque monkeys and assess whether neutralization of Nogo-A enhances motor recovery after spinal lesion in primates.

The motoneurons controlling hand muscles in macaque monkeys are located at the cervical and thoracic levels ${ }^{10}$ (Supplementary Fig. 1 online). We produced deficits in manual dexterity in 12 monkeys by unilateral cervical cord lesion at the C7-C8 border, rostral to the hand motoneurons (Supplementary Fig. 1, Supplementary Methods and Supplementary Table 1 online), aimed unilaterally at the dorsolateral funiculus, which was completely interrupted in eight monkeys. In four monkeys, the lesion was incomplete, with a few corticospinal axons preserved in the dorsolateral funiculus. The monkeys exhibited substantial postlesion loss of manual dexterity ipsilesionally, accompanied by hindlimb dysfunction.

The deficit in manual dexterity was followed by a progressive recovery. However, in a first 'pair' of monkeys (Fig. 1a) subjected to a large lesion, recovery was fast and complete in the Nogo-A-specific antibody-treated monkey (Mk-AM), in contrast to the control antibody-treated monkey ( $\mathrm{Mk}-\mathrm{CH})$. In a second pair of monkeys (Fig. 1b), the Nogo-A-specific antibody-treated monkey (Mk-AC) had a larger lesion but exhibited faster recovery than the control antibody-treated monkey (Mk-CG). Similarly, in a third pair of monkeys (Fig. 1c), the Nogo-A-specific antibody-treated monkey (Mk-AF) recovered better than the control antibody-treated monkey (Mk-CS). The functional recovery, plotted as a function of lesion extent (Fig. 1d), was significantly higher in the Nogo-A-specific antibody-treated monkeys than in control antibody-treated monkeys $(P=0.037)$. In monkeys treated with Nogo-A-specific antibody, the recovery was complete (or nearly so) irrespective of lesion extent, whereas in control-antibody treated monkeys there was an inverse correlation between lesion extent and functional recovery (Supplementary Fig. 1).

Based on a 'reach and grasp drawer' test (Supplementary Fig. 2 online) assessing ability to generate force with the fingers, the Nogo-A-specific antibody-treated monkeys tended to recover the capacity to pull the drawer faster than the control antibody-treated monkeys $(P=0.27)$, especially in case of large lesions (Fig. 2a). The difference was statistically significant when excluding the monkey $\mathrm{Mk}-\mathrm{CP}$, which had an incomplete lesion of the dorsolateral funiculus $(P=0.05)$. Whether and how a monkey could catch an object thrown to it ('ballistic arm movements' test; Supplementary Fig. 2) also indicated faster recovery of the capacity to preshape the hand (extension of fingers) in the Nogo-A-specific antibody-treated monkeys (Fig. 2b). This was a trend; however, it was not statistically significant $(P=0.12$ without monkey Mk-CP). We saw no difference between the two groups of monkeys regarding capacity of prehension with the hindlimb (Supplementary Fig. 2).

We labeled corticospinal axons by injecting the anterograde tracer biotinylated dextran amine (BDA) in the contralesional motor cortex (Supplementary Fig. 1). In the pair of monkeys Mk-CH and Mk-AM, corticospinal axonal arbors were more numerous caudal to the lesion in the Nogo-A-specific antibody-treated than in the control antibody-treated monkey (Fig. 2c,d). After normalization

${ }^{1}$ Unit of Physiology and Program in Neurosciences, Department of Medicine, Faculty of Sciences, University of Fribourg, Chemin du Musée 5, CH-1700 Fribourg, Switzerland. ${ }^{2}$ Brain Research Institute, University of Zürich, Department of Biology, ETH Zürich, Winterthurerstr. 190, CH-8057 Zürich, Switzerland. ${ }^{3}$ Department of Neurosurgery, Neurosurgery Clinic, University Hospital of Lausanne, Rue du Bugnon, $\mathrm{CH}-1011$ Lausanne, Switzerland. ${ }^{4}$ Neuroscience Research, Novartis Institute for BioMedical Research, CH-4002 Basel, Switzerland. ${ }^{5}$ These authors contributed equally to the study. Correspondence should be addressed to E.M.R. (Eric.Rouiller@unifr.ch). 


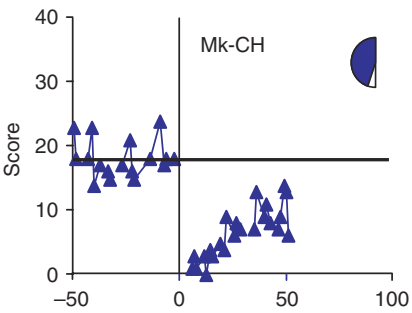

$\underline{\text { Nogo-A-specific antibody-treated monkeys }}$

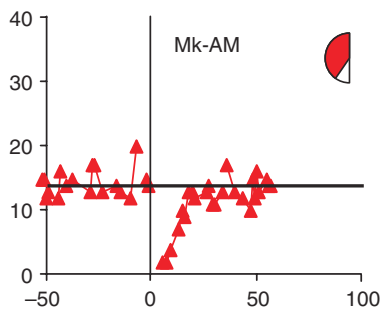

C
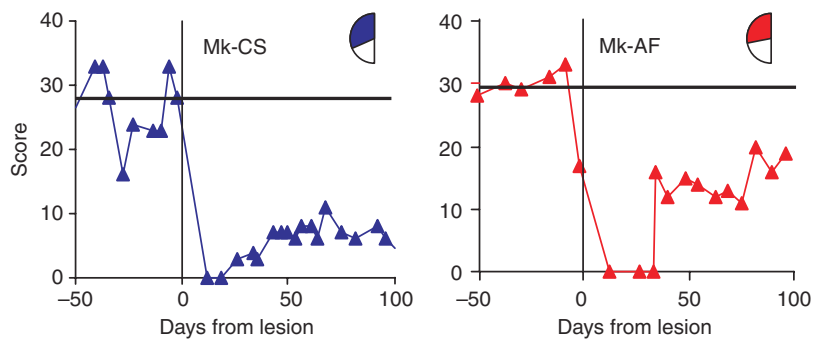

Control antibody-treated monkeys

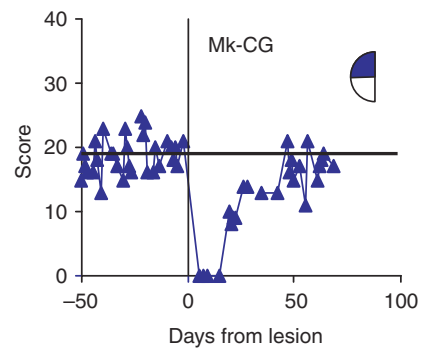

d

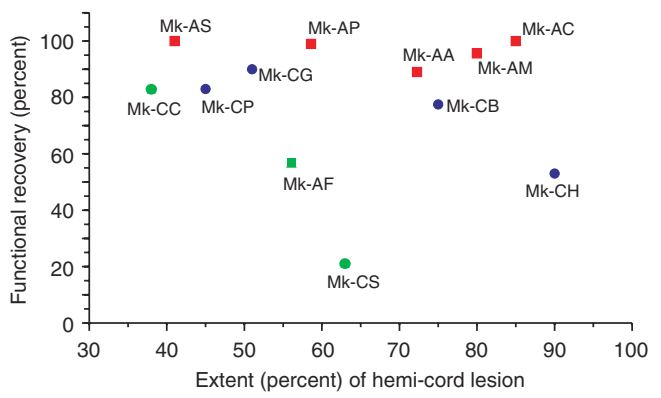

Figure 1 Quantitative assessment of manual dexterity before and after lesion. To reduce interindividual variability, we first performed direct comparisons between two monkeys, forming a 'pair' (Supplementary Methods online). (a-c) For three pairs of monkeys, comparison of behavioral scores assessing manual dexterity, before and after the lesion (day 0), derived from the 'modified Brinkman board' task (number of pellets retrieved within $30 \mathrm{~s}$ ) for the hand affected by the cervical lesion. The extent of the blue and red zones in the semicircular figures represents the extent of the hemicord lesion for each monkey (but does not indicate the location of the lesion). The two monkeys shown in c were subjected to intracortical stimulation (ICMS), possibly explaining the less prominent recovery than in the other monkeys. (d) Relationship between the extent of the hemicord lesion and the degree of functional recovery for the modified Brinkman board test. Blue circles represent control antibody-treated monkeys, red squares represent Nogo-A-specific antibody-treated monkeys. Three monkeys were plotted separately (green symbols) because they had been subjected to extensive ICMS ${ }^{14,15}$ which may have reduced their manual capacity. The recovery of manual dexterity was significantly higher (Mann-Whitney test, $P=0.028$ ) in the five Nogo-A-specific antibody-treated monkeys than in the four control antibody-treated monkeys. A statistically significant difference was also found when all 12 monkeys were included (Mann-Whitney test, $P=0.037$ ). (e) Left hand of a monkey performing the modified Brinkman board task, using the precision grip (opposition of thumb and index finger).

(Supplementary Table 1 and Supplementary Fig. 3 online), in six monkeys with complete corticospinal transection in which BDAlabeling data could be quantified caudal to the lesion, the normalized cumulated axonal arbor length was larger in the Nogo-A-specific antibody-treated than in the control antibody-treated monkeys (Fig. 2e). This was a trend; however, it was not statistically significant $(P=0.12)$. The normalized number of corticospinal axonal swellings caudal to the lesion was significantly larger (Fig. 2f) in the Nogo-Aspecific antibody-treated than in the control antibody-treated monkeys with complete lesion $(P<0.05$; if monkey Mk-CP is included, the difference is not statistically significant, $P=0.29$ ). Accordingly, the normalized number of axonal swellings plotted as a function of normalized axonal arbor length was higher in the Nogo-A-specific antibody-treated monkeys (Fig. 2g). We observed sprouting for a total distance of about $10-12 \mathrm{~mm}$ and it occurred from axotomized corticospinal axons (Supplementary Fig. 1) but sprouting from intact corticospinal axons from both hemispheres may also have occurred.

The monkeys treated with Nogo-A-specific antibody did not lose weight (Supplementary Fig. 3) and remained cooperative with the experimenter. Furthermore, the monkeys did not show signs of discomfort or pain when the experimenter touched their bodies or manipulated their limbs. These observations suggest that the rewiring induced by the Nogo-A-specific antibody treatment did not generate chronic pain (Supplementary Note online).

Neutralization of Nogo-A promotes regrowth of corticospinal (and possibly other) axons around the lesion and into the denervated spinal cord in macaque monkeys, in line with previous observation in marmosets 9 . For the first time in primates, Nogo-A-specific antibody treatment also led to considerable functional recovery. The time course and extent of spontaneous recovery of manual dexterity in the control antibody-treated monkeys are consistent with earlier reports for monkeys subjected to C3-C5 level lesion ${ }^{11-13}$. This time course was shortened and the recovery of skilled hand function was nearly complete when neutralizing Nogo-A (Figs. 1 and 2). Neutralization of Nogo-A by specific antibodies infused intrathecally led to extensive functional recovery and enhanced regenerative axonal sprouting and elongation not only in spinal cord-lesioned adult rats but also in adult primates, in the absence of detectable malfunctions. These observations represent an important proof of principle on the way to a new therapy of spinal cord and brain lesions in humans.

The experiments were carried out in accordance with the Guide for the Care and Use of Laboratory Animals and approved by local (Swiss) veterinary authorities.

Video sequences illustrating the behavioral tests used here can be seen at: http://www.unifr.ch/neuro/rouiller/motorcontcadre.htm. 
a

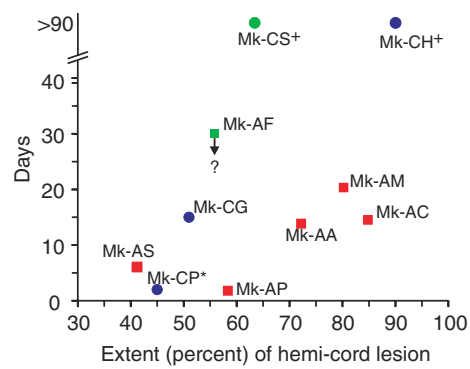

b

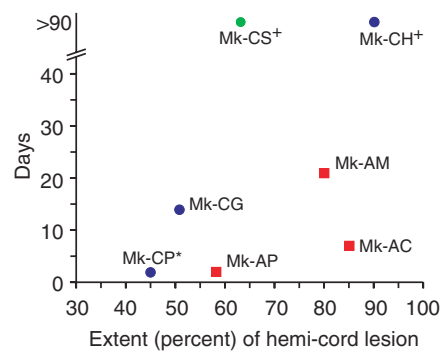

C

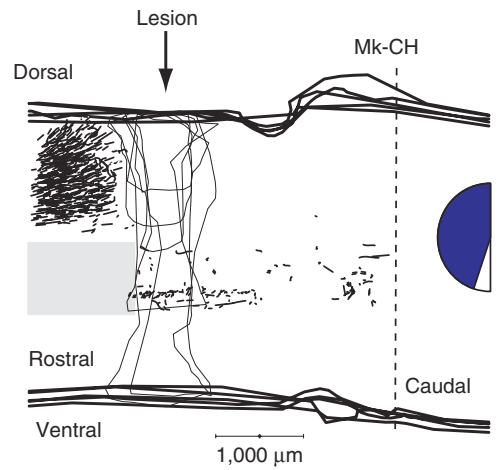

d

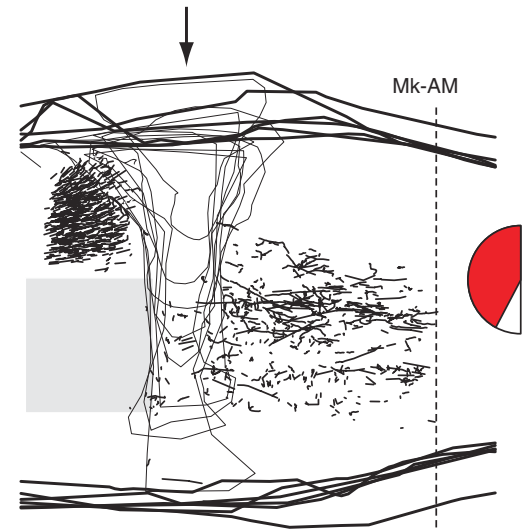

e

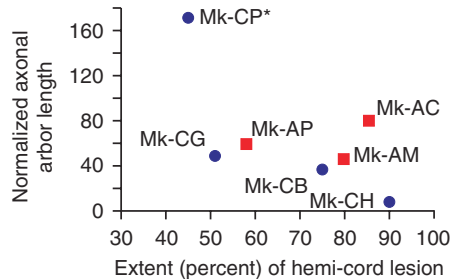

f

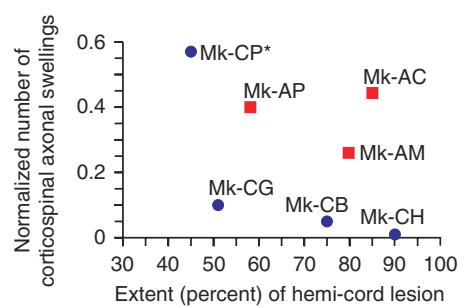

g

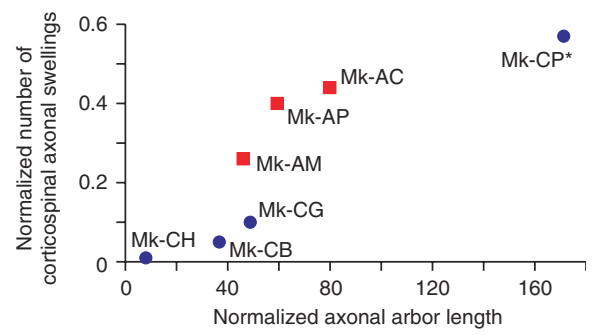

Figure 2 Nogo-A-specific antibody enhanced motor recovery and corticospinal axonal sprouting. Days required after lesion to recover the ability to perform the reach and grasp drawer (a) and ballistic arm movement (b) tasks plotted as a function of lesion extent. Monkey Mk-AF was not tested before $30 \mathrm{~d}$ for technical reasons. In monkey Mk-CP (indicated by an asterisk), the dorsolateral funiculus was not completely interrupted, possibly explaining the rapid recovery. Two monkeys did not recover during the 3 months of observation (indicated by plus signs). (c,d) Corticospinal axons were labeled by multiple, widespread BDA injections in the contralesional motor cortex. In two monkeys, in which all labeled corticospinal axons were interrupted, BDA-labeled axonal arbors caudal to the lesion were more numerous in the Nogo-A-specific antibody-treated (Mk-AM; d) than in the control antibody-treated monkey (Mk-CH; c). The gray area rostral to the lesion delineates the gray matter. In the seven monkeys for which we quantitatively analyzed BDA labeling caudal to the lesion, the normalized cumulative corticospinal axonal arbor length $(\mathrm{mm})$ and the normalized number of axonal swellings emitted by corticospinal axons (representing putative re-established contacts with interneurons or motoneurons deprived of inputs as a result of the lesion) were plotted as a function of lesion extent (e,f). Monkey Mk-CP, with incomplete section of the dorsolateral funiculus (indicated by an asterisk), had profuse sprouting from spared corticospinal axons. The number of corticospinal axonal swellings was plotted as a function of the cumulative corticospinal axonal arbor length (in mm) for the seven monkeys (g). Blue circles, control antibody-treated monkeys; red squares, Nogo-A-specific antibody-treated monkeys. Two monkeys were plotted separately (green symbols) because they had been subjected to extensive ICMS. The extent of the blue and red zones in the semicircular figures represents the extent of the hemicord lesion.

\section{ACKNOWLEDGMENTS}

The authors wish to thank the following for their technical assistance: G. Fischer, V. Moret, C. Roulin and F. Tinguely (histology and behavioral evaluations), J. Corpataux, B. Bapst and B. Morandi (animal house keeping), A. Gaillard (mechanics), B. Aebischer (electronics), L. Monney (informatics). This study was supported by the Swiss National Science Foundation grants 31-61857.00

(to E.M.R.), 31-63633.00 (to M.E.S.) and 4038043918/2 (PNR-38); the Novartis Foundation, the National Centre of Competence in Research on "Neural plasticity and repair," and the Christopher Reeves Foundation (Springfield, NJ). Antibodies were provided by Novartis Pharma.

\section{AUTHOR CONTRIBUTIONS}

P.F. contributed to behavioral data, tracing data and first draft of the manuscript. E.S. contributed to behavioral data, electrophysiology (ICMS), tracing data and study design. T.W. contributed to behavioral data, tracing data, electrophysiology (ICMS) and study design. J.B. contributed to the surgical procedure for hemicord lesion, placing the osmotic pumps and study design. A.M. contributed to study design and antibody protocol. M.E.S. contributed to study design and general concept, antibody protocol, data analysis and helped in the later phase of manuscript writing. E.M.R. contributed to study design, behavioral data, tracing data, electrophysiology (ICMS) and was primary author of the manuscript.

\section{COMPETING INTERESTS STATEMENT}

The authors declare competing financial interests (see the Nature Medicine website for details).

1. Filbin, M.T. Nat. Rev. Neurosci. 4, 703-713 (2003).

2. Schwab, M.E. Curr. Opin. Neurobiol. 14, 118-124 (2004).

3. Bregman, B.S. et al. Nature 378, 498-501 (1995).

4. Liebscher, T. et al. Ann. Neurol. 58, 706-719 (2005).

5. Nudo, R.J. \& Frost, S.B. in Evolution of Nervous Systems Vol 4 (eds. Krubitzer, L. \& Kaas, J.) (Elsevier Science, San Diego, 2006).

6. Lawrence, D.G. \& Hopkins, D.A. Brain 99, 235-254 (1976).

7. Porter, R. Electroencephalogr. Clin. Neurophysiol. 76, 282-293 (1990).

8. Lemon, R.N. Exp. Physiol. 78, 263-301 (1993).

9. Fouad, K., Klusman, I. \& Schwab, M.E. Eur. J. Neurosci. 20, 2479-2482 (2004).

10. Jenny, A.B. \& Inukai, J. J. Neurosci. 3, 567-575 (1983).

11. Galea, M.P. \& Darian-Smith, I. J. Comp. Neurol. 381, 282-306 (1997).

12. Galea, M.P. \& Darian-Smith, I. J. Comp. Neurol. 381, 307-319 (1997).

13. Sasaki, S. et al. J. Neurophysiol. 92, 3142-3147 (2004).

14. Schmidlin, E., Wannier, T., Bloch, J. \& Rouiller, E.M. Brain Res. 1017, 172-183 (2004).

15. Schmidlin, E. et al. BMC Neurosci. 6, 56 (2005) 